\title{
Characterization of generalized invexity in multi-objective fractional variational problem
}

\author{
Promila Kumar $^{1}$, Jyoti ${ }^{2, *}$, Bharti Sharma ${ }^{2}$ \\ ${ }^{1}$ Department of Mathematics, Gargi College, University of Delhi, Delhi 110049, India.
}

(Received: 10 March 2016; Accepted: 14 November 2016)

\begin{abstract}
In this article we define certain conditions on the functionals of multi-objective fractional variational problem in order that it becomes F-Kuhn Tucker pseudo invex or F-Fritz John pseudo invex. We also define F-KT and F-FJ points. Further, these problems are characterized such that all F-KT and F-FJ points become efficient solutions for the featured problem. An example is presented to verify the existence of F-KT point. A Parametric dual is proposed and various duality results are proved under the assumption of F-KT as well as F-FJ pseudo invexity.
\end{abstract}

Keywords Multi-objective, Fractional, Variational Problem, Pseudoinvexity, Efficient Solution, F-Fritz John Point, FKuhn-Tucker Point

AMS 2010 subject classifications 90C46, 90C29

DOI: $10.19139 /$ soic.v4i4.208

\section{Introduction}

The problem of finding a function for which the value of a certain integral over an interval of time, technically known as the functional, is either the largest or smallest possible subject to certain constraints is termed as Constrained Variational problem.

The relationship between mathematical programming problem and classic calculus of variations was explored by Hanson [13]. After that, there has been a significant growth in the study of optimality and duality conditions for variational /control problems. Several contributions have been made to its development by researchers, see $[4,5,6,1,18,17]$ and the references therein.

Hanson gave the concept of Invexity [12] which was extended to functionals by Mond and Smart [2] . Bhatia and Kumar [4] used generalized invexity to establish optimality and duality for control problems. Recently Kumar and Jyoti [15] proposed generalized invexity of higher order for functionals and applied this to study variational problems. Kumar and Sharma [16] used weak efficiency of higher order to establish relationship between primal and dual for multi-objective fractional variational problem.

Invexity defined by Hanson [12] is a sufficient condition but not a necessary condition for every Kuhn Tucker stationary point to be a global minimizer for constrained problems. Martin [7] defined KT-invexity for scalar minimization problem and characterized these functions in order that all Kuhn Tucker point are global minimizer. Arana et al. [10] extended these results to a continuous case and introduced L-KT pseudoinvexity for single objective variational problem. Their results were extended to multi-objective variational problem-'the problem of simultaneously optimizing several objective functionals which are conflicting in nature', by Jimenez [14] and

\footnotetext{
${ }^{*}$ Correspondence to: Jyoti (Email: deepshahj_p@yahoo.co.in). Department of Mathematics, University of Delhi, Delhi 110007, India.
}

ISSN 2310-5070 (online) ISSN 2311-004X (print)

Copyright (C) 2016 International Academic Press 
they named it as V-KT pseudoinvexity. These problems become all the more important when the objective function is a ratio of two functionals. Such problems are termed as multi-objective fractional variational problem. The presence of multi-objective fractional variational problem is felt prominently due to its applications in wide variety of fields such as management science, economics, engineering problems, industrial process control, production and inventory and many more. This motivates us to propose the notion of F-KT invexity for such problems.

In this article, we define certain conditions on functionals of multi-objective fractional variational problem in order that it becomes F-KT pseudo invex or F-FJ pseudo invex. We also define F-KT and F-FJ points. Further, these problems are characterized such that all F-KT and F-FJ points become efficient solutions for the featured problem. An example is presented to verify the existence of F-KT point. A Parametric dual is proposed and various duality results are proved under the assumption of F-KT as well as F-FJ pseudo invexity.

\section{Definitions and preliminaries}

Let $\mathbb{R}^{n}$ denotes a n-dimensional Euclidean space. Let $r, n$ and $p$ be three positive integers. For a given real interval $I=[a, b]$, let $f^{i}: I \times \mathbb{R}^{n} \times \mathbb{R}^{n} \rightarrow \mathbb{R}, i=1,2, \ldots, r, k^{i}: I \times \mathbb{R}^{n} \times \mathbb{R}^{n} \rightarrow \mathbb{R}, i=1,2, \ldots, r$ and $g^{j}: I \times \mathbb{R}^{n} \times$ $\mathbb{R}^{n} \rightarrow \mathbb{R}, j=1,2, \ldots, p$ be continuously differentiable functions with respect to each of their arguments.

Let $x: I \rightarrow \mathbb{R}^{n}$ be a piecewise smooth state function with its derivative $\dot{x}$. For notational convenience $x(t)$ and $\dot{x}(t)$ will be written as $x$ and $\dot{x}$ respectively. Denote the partial derivative of $f^{i}, i=1,2, \ldots, r$, with respect to $t, x$ and $\dot{x}$ by $f_{t}^{i}, f_{x}^{i}, f_{\dot{x}}^{i}$ respectively. Analogously, we write the partial derivative of $k^{i}$ and $g^{j}$.

For any $x=\left(x^{1}, x^{2}, \ldots, x^{n}\right)^{T}, y=\left(y^{1}, y^{2}, \ldots, y^{n}\right)^{T}$, We define

(i) $x<y \Leftrightarrow x^{i}<y^{i}$ for all $i=1,2, \ldots, n$.

(ii) $x \leqq y \Leftrightarrow x^{i} \leq y^{i}$ for all $i=1,2, \ldots, n$.

(iii) $x \leq y \Leftrightarrow x \leqq y$ and $x \neq y$.

Let $\mathbb{R}_{+}^{n}$ be nonnegative orthant of $\mathbb{R}^{n}$ and int $\mathbb{R}_{+}^{n}$ be positive orthant of $\mathbb{R}^{n}$. Let $X$ be the space of piecewise smooth state functions $x: I \rightarrow \mathbb{R}^{n}$ equipped with the norm $\|x\|=\|x\|_{\infty}+\|D x\|_{\infty}$ where the differential operator $D$ is given by $u=D x \Leftrightarrow x(t)=x(a)+\int_{a}^{t} u(s) d s$. Therefore, $D=\frac{d}{d t}$ except at discontinuities.

Now, consider the following multi-objective fractional variational problem (MFVP):

$$
\operatorname{Minimize}\left(\frac{\int_{a}^{b} f^{1}(t, x, \dot{x}) d t}{\int_{a}^{b} k^{1}(t, x, \dot{x}) d t}, \frac{\int_{a}^{b} f^{2}(t, x, \dot{x}) d t}{\int_{a}^{b} k^{2}(t, x, \dot{x}) d t}, \ldots, \frac{\int_{a}^{b} f^{r}(t, x, \dot{x}) d t}{\int_{a}^{b} k^{r}(t, x, \dot{x}) d t}\right)
$$

subject to

$$
\begin{gathered}
g(t, x, \dot{x})=\left(g^{1}(t, x, \dot{x}), g^{2}(t, x, \dot{x}), \ldots, g^{p}(t, x, \dot{x})\right) \leqq 0, t \in I \\
x(a)=\alpha, x(b)=\beta, \alpha, \beta \in \mathbb{R}^{n} .
\end{gathered}
$$

Assume that $\int_{a}^{b} f^{i}(t, x, \dot{x}) d t \geq 0$ and $\int_{a}^{b} k^{i}(t, x, \dot{x}) d t>0$ for all $i \in\{1,2, \ldots r\}$ and for all $x \in X$.

Let $X_{0}=\{x \in X \mid g(t, x, \dot{x}) \leqq 0, t \in I, x(a)=\alpha, x(b)=\beta\}$ be the set of feasible solutions of (MFVP).

Define

$$
\Phi(x)=\left(\frac{\int_{a}^{b} f^{1}(t, x, \dot{x}) d t}{\int_{a}^{b} k^{1}(t, x, \dot{x}) d t}, \frac{\int_{a}^{b} f^{2}(t, x, \dot{x}) d t}{\int_{a}^{b} k^{2}(t, x, \dot{x}) d t}, \ldots, \frac{\int_{a}^{b} f^{r}(t, x, \dot{x}) d t}{\int_{a}^{b} k^{r}(t, x, \dot{x}) d t}\right) .
$$

Arana et al. [9] proposed L-KT pseudoinvexity and L-FJ pseudoinvexity for a scalar variational problem. They [11] further generalized these concepts to vector variational problems. We take a step forward and propose F-KT pseudoinvexity and F-FJ pseudoinvexity for a multi-objective fractional variational problem. In the sequel, we give the definitions of efficient solution, F-Kuhn Tucker point and F-Fritz John point for (MFVP). 


\section{Definition 1}

$\bar{x} \in X_{0}$ is said to be an efficient solution for (MFVP) if there is no $x \in X_{0}$ such that

$$
\Phi(x) \leq \Phi(\bar{x})
$$

i.e.

$$
\begin{gathered}
\frac{\int_{a}^{b} f^{i}(t, x, \dot{x}) d t}{\int_{a}^{b} k^{i}(t, x, \dot{x}) d t} \leq \frac{\int_{a}^{b} f^{i}(t, \bar{x}, \dot{\bar{x}}) d t}{\int_{a}^{b} k^{i}(t, \bar{x}, \dot{\bar{x}}) d t}, \text { for all } i \in\{1,2, \ldots, r\} \text { and } \\
\frac{\int_{a}^{b} f^{j}(t, x, \dot{x}) d t}{\int_{a}^{b} k^{j}(t, x, \dot{x}) d t}<\frac{\int_{a}^{b} f^{j}(t, \bar{x}, \dot{\bar{x}}) d t}{\int_{a}^{b} k^{j}(t, \bar{x}, \dot{\bar{x}}) d t}, \text { for at least one } j \in\{1,2, \ldots, r\} .
\end{gathered}
$$

Definition 2

$\bar{x} \in X_{0}$ is said to be F-Kuhn Tucker point for (MFVP) if there exist $\bar{\lambda} \in \mathbb{R}^{r}, \bar{v} \in \mathbb{R}^{r}$ and a piecewise smooth function $y: I \longrightarrow \mathbb{R}^{p}$ such that

$$
\begin{gathered}
\sum_{i=1}^{r} \bar{\lambda}^{i}\left(f_{\bar{x}}^{i}(t)-\bar{v}^{i} k_{\bar{x}}^{i}(t)\right)+y(t)^{T} g_{\bar{x}}(t)=\frac{d}{d t}\left[\sum_{i=1}^{r} \bar{\lambda}^{i}\left(f_{\dot{\bar{x}}}^{i}(t)-\bar{v}^{i} k_{\dot{\bar{x}}}^{i}(t)\right)+y(t)^{T} g_{\dot{\bar{x}}}(t)\right], t \in I, \\
y(t)^{T} g(t, \bar{x}, \dot{\bar{x}}) d t=0,
\end{gathered}
$$$$
(\bar{\lambda}, \bar{v}, y(t)) \geqq 0, \bar{\lambda} \neq 0, \forall t \in I \text { except at discontinuities. }
$$

Remark 3

$$
\text { If }(\bar{\lambda}, \bar{v}, y(t)) \geqq 0,(\bar{\lambda}, y(t)) \neq 0,
$$

holds instead of (5), then $\bar{x}$ is said to be F-Fritz John point for (MFVP).

The example given below ensures the existence of F-Kuhn Tucker Points for (MFVP).

Example 4

$$
(P 1): \operatorname{Minimize}\left(\frac{\int_{0}^{1}\{x(t)(x(t)-t(t-1))\}^{2} d t}{\int_{0}^{1}\left\{x^{2}(t)+1\right\} d t}, \frac{\int_{0}^{1}\left\{\dot{x}(t)\left(t^{2}+2\right)\right\}^{2} d t}{\int_{0}^{1}\left\{\dot{x}^{2}(t)+1\right\} d t}\right)
$$

subject to

$$
\begin{aligned}
& -x(t)+\dot{x}(t) \leq 1 \\
& x(0)=0, x(1)=0 .
\end{aligned}
$$

Then $\bar{x}(t)=0, t \in[0,1]$ is a F-Kuhn Tucker point for $(\mathrm{P} 1)$, as there exist $\bar{\lambda}=\left(\frac{1}{2}, \frac{1}{2}\right) \in \mathbb{R}^{2}, \bar{v}=(0,0)$ and smooth function $y: I \rightarrow \mathbb{R}$,such that $y(t)=0$, for $t \in I$. It is easy to verify that (3), (4), and (5) are satisfied.

Definition 5

The problem (MFVP) is said to be F-KT pseudoinvex at $\bar{x} \in X_{0}$ if given $x \in X, \lambda \in \mathbb{R}^{r}, v \in \mathbb{R}^{r}$ and a piecewise smooth function $y: I \longrightarrow \mathbb{R}^{p}$, where $(\bar{x}, \lambda, v, y)$ satisfies (4) and (5), and there exists a differentiable function $\eta(t, x, \bar{x}, \lambda, v, y): I \longrightarrow \mathbb{R}^{n}$ with $\eta(a, x, \bar{x}, \lambda, v, y)=0=\eta(b, x, \bar{x}, \lambda, v, y)$ such that 


$$
\begin{gathered}
\Phi(x) \leq \Phi(\bar{x}) \Rightarrow \\
H(\eta(t, x, \bar{x}, \lambda, v, y))=\int_{a}^{b}\left\{\left[\sum_{i=1}^{r} \bar{\lambda}^{i}\left(f_{\bar{x}}^{i}(t)-\bar{v}^{i} k_{\bar{x}}^{i}(t)\right)+y(t)^{T} g_{\bar{x}}(t)\right] \eta(t, x, \bar{x}, \lambda, v, y)\right. \\
\left.+\left[\sum_{i=1}^{r} \bar{\lambda}^{i}\left(f_{\overline{\bar{x}}}^{i}(t)-\bar{v}^{i} k_{\overline{\bar{x}}}^{i}(t)\right)+y(t)^{T} g_{\dot{\bar{x}}}(t)\right] \frac{d}{d t} \eta(t, x, \bar{x}, \lambda, v, y)\right\} d t<0 .
\end{gathered}
$$

\section{Remark 6}

If $(\bar{x}, \lambda, v, y)$ satisfies $(4)$ and $(6)$, other conditions being the same as the previous definition then the problem (MFVP) is said to be F-FJ pseudoinvex at $\bar{x} \in X_{0}$.

Remark 7

It is easy to prove that if (MFVP) is F-KT pseudoinvex, then (MFVP) is F-FJ pseudoinvex.

The following Lemma [19] is required to prove the optimality conditions in the proceeding section.

\section{Lemma 1}

Let $\bar{x}$ be a normal efficient solution for (MFVP). Then $\bar{x}$ is a F-Kuhn-Tucker point.

\section{Characterization of F-Pseudoinvexity}

In this section we characterize the multi-objective fractional variational problem and establish optimality for (MFVP).

\section{Theorem 8}

If all F-Kuhn-Tucker point are efficient solution for (MFVP), then (MFVP) is F-KT pseudoinvex on $X_{0}$.

Proof

Let us assume that all F-KT points of (MFVP) are efficient solutions of (MFVP). Let $x, \bar{x} \in X_{0}, \lambda, v \in \mathbb{R}^{r}$ and $y: I \rightarrow \mathbb{R}^{p}$ be a piecewise smooth function such that $(\bar{x}, \lambda, v, y)$ satisfies $(4)$ and (5)

$$
\Phi(x) \leq \Phi(\bar{x})
$$

To prove that (MFVP) is F-KT pseudoinvex on $X_{0}$, we have to show that the existence of some differentiable function $\eta$ with $\eta(a, x, \bar{x}, \lambda, v, y)=0=\eta(b, x, \bar{x}, \lambda, v, y)$ such that $H(\eta(t, x, \bar{x}, \lambda, v, y))<0$.

On the contrary, assume that there is no such $\eta(t, x, \bar{x}, \lambda, y)$ for which $H(\eta(t, x, \bar{x}, \lambda, v, y))<0$.

Then it is trivial that there is no such $\eta(t, x, \bar{x}, \lambda, y)$ for which $H(\eta(t, x, \bar{x}, \lambda, v, y))>0$.

Therefore $H(\eta(t, x, \bar{x}, \lambda, v, y))=0$ for all $\eta(t, x, \bar{x}, \lambda, v, y)$ with $\eta(a, x, \bar{x}, \lambda, v, y)=0=\eta(b, x, \bar{x}, \lambda, v, y)$. which implies

$$
\begin{gathered}
\int_{a}^{b}\left(\left(\sum_{i=1}^{r} \bar{\lambda}^{i}\left(f_{\bar{x}}^{i}(t)-\bar{v}^{i} k_{\bar{x}}^{i}(t)\right)+y(t)^{T} g_{\bar{x}}(t)\right) \eta(t, x, \bar{x}, \lambda, v, y)-\frac{d}{d t}\left(\sum_{i=1}^{r} \bar{\lambda}^{i}\left(f_{\overline{\bar{x}}}^{i}(t)-\bar{v}^{i} k_{\overline{\bar{x}}}^{i}(t)\right)+y(t)^{T} g_{\dot{\bar{x}}}(t)\right) \eta(t, x, \bar{x}, \lambda, v, y)\right) d t \\
\left.+\sum_{i=1}^{r} \bar{\lambda}^{i}\left(f_{\bar{x}}^{i}(t)-\bar{v}^{i} k_{\bar{x}}^{i}(t)\right)+y(t)^{T} g_{\bar{x}}(t)\right)\left.\eta(t, x, \bar{x}, \lambda, v, y)\right|_{a} ^{b}=0
\end{gathered}
$$

for all $\eta(t, x, \bar{x}, \lambda, v, y)$ with $\eta(a, x, \bar{x}, \lambda, v, y)=0=\eta(b, x, \bar{x}, \lambda, v, y)$.( By using integration by parts)

By Fundamental theorem of Calculus of Variation, [8] we have

$$
\sum_{i=1}^{r} \bar{\lambda}^{i}\left(f_{\bar{x}}^{i}(t)-\bar{v}^{i} k_{\bar{x}}^{i}(t)\right)+y(t)^{T} g_{\bar{x}}(t)=\frac{d}{d t}\left[\sum_{i=1}^{r} \bar{\lambda}^{i}\left(f_{\bar{x}}^{i}(t)-\bar{v}^{i} k_{\overline{\bar{x}}}^{i}(t)\right)+y(t)^{T} g_{\overline{\bar{x}}}(t)\right]
$$


Therefore $(\bar{x}, \lambda, v, y)$ satisfies $(3),(4),(5)$.

This implies that $\bar{x}$ is a F-Kuhn Tucker point of (MFVP).

By virtue of the hypothesis, all F-KT points, in particular $\bar{x}$ is an efficient solution of (MFVP).

This contradicts

$$
\Phi(x) \leq \Phi(\bar{x}) .
$$

Hence (MFVP) is F-KT pseudoinvex on $X_{0}$.

Theorem 9

If (MFVP) is F-KT pseudoinvex on $X_{0}$, then all F-Kuhn Tucker point for (MFVP) are efficient solution for (MFVP).

Proof

Let $\bar{x} \in X_{0}$ be a F-Kuhn Tucker point then there exist $\bar{\lambda}, \bar{v} \in \mathbb{R}^{r}$ and piecewise smooth function $y: I \rightarrow \mathbb{R}^{p}$ such that $(\bar{x}, \bar{\lambda}, \bar{v}, y)$ satisfies $(3),(4),(5)$. Let $x \in X_{0}$, since (MFVP) is F-KT pseudoinvex, there exists a differentiable function $\eta$ with

$$
\eta(a, x, \bar{x}, \lambda, v, y)=0=\eta(b, x, \bar{x}, \lambda, v, y)
$$

such that whenever

$$
\Phi(x) \leq \Phi(\bar{x})
$$

$\Rightarrow H(\eta(t, x, \bar{x}, \lambda, v, y))<0$.

Integrating by parts and using equations (3) and (7), we get $H(\eta(t, x, \bar{x}, \lambda, v, y))=0$, which is a contradiction Hence there is no $x \in X_{0}$ such that

$$
\Phi(x) \leq \Phi(\bar{x}) .
$$

It implies that $\bar{x}$ is an efficient solution for (MFVP).

Theorem 10

All F-Fritz John point for (MFVP) are efficient solution for (MFVP) iff (MFVP) is F-FJ pseudoinvex.

Proof

Proof runs on the same lines as that of above two theorems.

\section{Duality}

Following the parametric approach of Bector et al.[3], we now present the following Modified dual to (MFVP), (MFVD) Maximize $\bar{v}=\left(\bar{v}^{1}, \ldots, \bar{v}^{r}\right)$

subject to

$$
\begin{gathered}
\sum_{i=1}^{r} \bar{\lambda}^{i}\left(f_{\bar{x}}^{i}(t)-\bar{v}^{i} k_{\bar{x}}^{i}(t)\right)+y(t)^{T} g_{\bar{x}}(t)=\frac{d}{d t}\left[\sum_{i=1}^{r} \bar{\lambda}^{i}\left(f_{\overline{\bar{x}}}^{i}(t)-\bar{v}^{i} k_{\overline{\bar{x}}}^{i}(t)\right)+y(t)^{T} g_{\dot{\bar{x}}}(t)\right], t \in I, \\
y(t)^{T} g(t, \bar{x}, \dot{\bar{x}}) d t=0, \\
\int_{a}^{b}\left(f^{i}(t, \bar{x}, \dot{\bar{x}})-\bar{v}^{i} k^{i}(t, \bar{x}, \dot{\bar{x}}) d t \geq 0, i=1,2, \ldots, r,\right.
\end{gathered}
$$




$$
\bar{x} \in X, v^{i} \geq 0, \bar{\lambda} \geq 0, i=1,2, \ldots, r, \sum_{i=1}^{r} \lambda^{i}=1, y(t) \geqq 0, t \in I .
$$

Let $Y_{1}$ be the set of all feasible solutions of (MFVD)

\section{Theorem 11}

(Weak duality) Let $x \in X_{0}$ and $(\bar{x}, \bar{\lambda}, \bar{v}, y) \in Y_{1}$. If (MFVP) is F-KT pseudoinvex at $\bar{x}$. Then the following cannot hold

$$
\begin{gathered}
\frac{\int_{a}^{b} f^{i}(t, x, \dot{x}) d t}{\int_{a}^{b} k^{i}(t, x, \dot{x}) d t} \leq \bar{v}^{i}, \text { for all } i \in\{1,2, \ldots, r\} \text { and } \\
\frac{\int_{a}^{b} f^{j}(t, x, \dot{x}) d t}{\int_{a}^{b} k^{j}(t, x, \dot{x}) d t}<\bar{v}^{j}, \text { for at least one } j \in\{1,2, \ldots, r\} .
\end{gathered}
$$

Proof

On the contrary, assume that inequalities (12) and (13) holds. It follows from (10) that

$$
\begin{gathered}
\frac{\int_{a}^{b} f^{i}(t, x, \dot{x}) d t}{\int_{a}^{b} k^{i}(t, x, \dot{x}) d t} \leq \frac{\int_{a}^{b} f^{i}(t, \bar{x}, \dot{\bar{x}}) d t}{\int_{a}^{b} k^{i}(t, \bar{x}, \dot{\bar{x}}) d t} \text { for all } i \in\{1,2, \ldots, r\} \text { and } \\
\frac{\int_{a}^{b} f^{j}(t, x, \dot{x}) d t}{\int_{a}^{b} k^{j}(t, x, \dot{x}) d t}<\frac{\int_{a}^{b} f^{j}(t, \bar{x}, \dot{\bar{x}}) d t}{\int_{a}^{b} k^{j}(t, \bar{x}, \dot{\bar{x}}) d t}, \text { for at least one } j \in\{1,2, \ldots, r\} .
\end{gathered}
$$

Since $(\bar{x}, \bar{\lambda}, \bar{v}, y)$ satisfies (9) and (11) and (MFVP) is F-KT pseudoinvex at $\bar{x}$, there exists a differentiable function $\eta$ with

$$
\eta(a, x, \bar{x}, \lambda, v, y)=0=\eta(b, x, \bar{x}, \lambda, v, y)
$$

such that $H(\eta(t, x, \bar{x}, \lambda, v, y))<0$.

Integrating by parts and using equations (8) and (14),we get $H(\eta(t, x, \bar{x}, \lambda, v, y))=0$.

But this is a contradiction to the above inequality.

Hence the results follows.

\section{Theorem 12}

(Strong duality) Let $\bar{x}$ be normal efficient solution of (MFVP). There exists $\bar{\lambda}, \bar{v} \in R^{r}$ and piecewise smooth function $y: I \longrightarrow R^{p}$ such that $(\bar{x}, \bar{\lambda}, \bar{v}, y) \in Y_{1}$. If (MFVP) is F-KT pseudoinvex, then $(\bar{x}, \bar{\lambda}, \bar{v}, y)$ is an efficient solution for (MFVD).

\section{Proof}

Since $\bar{x}$ is a normal efficient solution for (MFVP), then by lemma 1, it follows that $\bar{x}$ is a F-Kuhn Tucker point. Then there exists $\bar{\lambda}, \bar{v} \in R^{r}$ and piecewise smooth function $y: I \longrightarrow R^{p}$ such that $(\bar{x}, \bar{\lambda}, \bar{v}, y) \in Y_{1}$. Suppose $(\bar{x}, \bar{\lambda}, \bar{v}, y) \in Y_{1}$ is not an efficient solution for (MFVP), then there exists $(\hat{x}, \hat{v}, \hat{\lambda}, \hat{y})$ such that

$$
\begin{gathered}
\bar{v} \leqslant \hat{v} \\
\frac{\int_{a}^{b} f^{i}(t, \bar{x}, \dot{\bar{x}}) d t}{\int_{a}^{b} k^{i}(t, \bar{x}, \dot{\bar{x}}) d t}=\bar{v}^{i} \leqslant \hat{v}^{i} \leqslant \frac{\int_{a}^{b} f^{i}(t, \hat{x}, \dot{\hat{x}}) d t}{\int_{a}^{b} k^{i}(t, \hat{x}, \dot{\hat{x}}) d t} \text { for all } i \in\{1,2, \ldots, r\} \text { and }
\end{gathered}
$$




$$
\frac{\int_{a}^{b} f^{j}(t, \bar{x}, \dot{\bar{x}}) d t}{\int_{a}^{b} k^{j}(t, \bar{x}, \dot{\bar{x}}) d t}=\bar{v}^{j}<\hat{v}^{j} \leqslant \frac{\int_{a}^{b} f^{j}(t, \hat{x}, \dot{\hat{x}}) d t}{\int_{a}^{b} k^{j}(t, \hat{x}, \dot{\hat{x}}) d t} \text { for at least one } j \in\{1,2, \ldots, r\} .
$$

But this is a contradiction to weak duality theorem.

Hence $(\bar{x}, \bar{\lambda}, \bar{v}, y)$ is an efficient solution for (MFVD).

Theorem 13

(Converse Duality) Let $(\bar{x}, \bar{\lambda}, \bar{v}, y)$ be an efficient solution for (MFVD). If $\bar{x} \in X_{0}$ and (MFVP) is F-KT pseudoinvex. Then $\bar{x}$ is an efficient solution for (MFVP).

Proof

Suppose $\bar{x}$ is not an efficient solution for (MFVP), then there exists $\hat{x} \in X_{0}$ such that

$$
\Phi(\hat{x}) \leq \Phi(\bar{x}) .
$$

Also (MFVP) is F-KT pseudoinvex at $\bar{x}$, there exists a differentiable function $\eta$ with

$$
\eta(a, \hat{x}, \bar{x}, \lambda, v, y)=0=\eta(b, \hat{x}, \bar{x}, \lambda, v, y)
$$

such that $H(\eta(t, \hat{x}, \bar{x}, \lambda, v, y))<0$.

Integrating by parts and using equations (8) and (15),we get

$H(\eta(t, \hat{x}, \bar{x}, \lambda, v, y))=0$ which leads a contradiction to the above inequality.

Hence there is no $x \in X_{0}$ such that

$$
\Phi(x) \leq \Phi(\bar{x})
$$

Hence $\bar{x}$ is an efficient solution for (MFVP).

\section{Remark 14}

Similarly weak duality, strong duality and converse duality results are proved on the same lines as of the above theorem under the assumptions of F-FJ pseudoinvexity.

\section{Conclusion}

We focus on Multi-objective Fractional Variational Problem in this article. Certain conditions are imposed on the functionals of (MFVP) in order that it becomes F-Kuhn Tucker pseudo invex or F-Fritz John pseudo invex problem. F-KT point and F-FJ points are also defined for (MFVP). To authenticates the existence of F-KT point an example is presented in Section 2 of the paper. The Multi-objective Fractional Variational Problem is characterised such that 'the problem is F-KT(F-FJ) pseudo invex if and only if all F-KT(F-FJ) points are efficient solutions of the problem'. Finally, a parametric dual of (MFVP) is proposed for which weak, strong and converse duality theorems are established.

\section{Acknowledgement}

The authors are grateful to Professor (Mrs.) Davinder Bhaita (Rtd.) Dept. of Operational research for her kind guidance throughout the preparation of this paper. The author named Jyoti was supported by University Grant Commission Non-NET research fellowship, India (Grant no Non-NET/139/Ext-136/2014).

The author named Bharti sharma was supported by Council Of Scientific And Industrial Research, Junior Research Fellowship, India (Grant no 09/045(1350)/2014-EMR-1). The author are also thankful to anonymous referees for their valuable suggestions. 


\section{REFERENCES}

1. B. Mond, and M. A. Hanson, Duality for variational problem, Journal of Mathematical Analysis and Applications, vol. 18, pp. 355364, (1967).

2. B. Mond, and I. Smart, Duality and sufficiency in control problems with invexity, Journal of Mathematical Analysis and Applications, vol. 136, pp. 325-333 (1988).

3. C. R. Bector, S. Chandra, and I. Husain, Optimality condition and sub-differentiable multi-objective fractional programming, Journal of Optimization theory and Applications, vol. 79, pp.105-125, (1988) .

4. D. Bhatia, and P. Kumar, Multi-objective control problem with generalized invexity, Journal of Mathematical Analysis and Applications, vol. 189, pp.676-692, (1995).

5. D. Bhatia, and P. Kumar, On multi-objective fractional control problems, Journal of Operational Research, vol. 13, pp.115-132, (1996).

6. D. Bhatia, and P. Kumar, Duality for variational problems with b-vex functions, Optimization, vol. 36, pp.347-360, (1996).

7. D. H. Martin, The essence of invexity, Journal of Optimization Theory and Applications, vol. 47, pp. 65-76, (1985).

8. I. M. Gelfand, and S. V. Fomin, Calculas of Variation, Prentice hall, Inc. Englewood Cliff, New Jersy pp. 34-35, (1963).

9. M. Arana, R. Osuna, G. Ruiz, and M. Rojas, On variational problems: Characterization of solutions and duality, Journal of Mathematical Analysis and Applications, vol. 311, pp. 1-12, (2005).

10. M. Arana, A. Rufian, R. Osuna, and G. Ruiz, Pseudoinvexity, optimality conditions and efficiency in multi-objective problems; duality, Nonlinear Analysis, vol. 68, pp. 24-34, (2008).

11. M. Arana, G. Ruiz,A. Rufian, and R. Osuna, Weak efficiency in multi-objective variational problems under generalized convexity, Journal Of Global Optimization, vol. 52, pp. 109-121, (2012).

12. M. A. Hanson, On Sufficiency of Kuhn Tucker conditions, Journal of Mathematical Analysis and Applications vol. 80, pp.545-550, (1981).

13. M. A. Hanson, Bounds for functionally convex optimal control problems, Journal of Mathematical Analysis and Applications, vol. 8, pp.84-89,(1964).

14. M. A. Jimenez, G. R. Garzon, A. R. Lizana, and R. O. Gomez, A necessary and sufficient condition for duality in multi-objective variational problems, European Journal of Operational Research, vol. 201, pp.672-681,(2010).

15. P. Kumar, and Jyoti, Generalized invexity of higher order and its applications in variational problems, Applied Mathematics, vol. 6, No. 9, pp.1638-1648, (2015)

16. P. Kumar, and B. Sharma, Weak efficiency of higher order for multi-objective fractional variational problems, Opsearch, vol. 53, No. 3, pp.538-552, (2016).

17. R. Patel, Mixed type duality for multi-objective fractional variational problems using $(b, F, \rho)$ convexity, Journal of Combinatorics, Information ans System Sciences, vol. 35, No. 1/2, pp.59-79, (2010).

18. S.K.Mishra, and R.N.Mukherjee, Duality for multi-objective fractional variational problems, Journal of Mathematical Analysis and Applications, vol. 186, pp.711-725, (1994).

19. S. Mititelu, and I.M.Stancu-Minasian, Efficiency and duality for multi-objective fractional variational problems with $(\rho, b)$ quasiinvexity, Yogoslav Journal of Operations Research, vol. 19, pp.85-99, (2009). 